\title{
Neglect Performance in Acute Stroke Is Related to Severity of White Matter Hyperintensities
}

\author{
Zainab S. Bahrainwala ${ }^{a}$ Argye E. Hillis ${ }^{b}$ Jennifer Dearborn ${ }^{b}$ \\ Rebecca F. Gottesman ${ }^{b}$ \\ a School of Public Health, University of Medicine and Dentistry of New Jersey, Piscataway, N.J., and \\ ${ }^{b}$ Department of Neurology, Johns Hopkins University School of Medicine, Baltimore, Md., USA
}

\section{Key Words}

Leukoaraiosis · Neglect · Cognition · Stroke

\begin{abstract}
Background: Leukoaraiosis and its progression have longitudinally been associated with cognitive decline and dementia. Its role in acute cognitive function and response to acute cerebral ischemia is less well understood. We evaluated whether the presence and extent of leukoaraiosis, or white matter hyperintensities (WMH), had an impact on performance on tests of hemispatial neglect in acute ischemic stroke patients. Methods: A series of 206 acute ischemic right-hemispheric stroke patients at Johns Hopkins Hospital underwent brain MRI and cognitive assessment for hemispatial neglect within 5 days of symptom onset. Error rates on neglect tests were evaluated, as were dichotomized measures of neglect, including 'any', 'severe' or 'worst' neglect, based on Z scores of at least 2 on 1, 2 or 3 tests (respectively) within a neglect battery. Acute infarct volumes were measured on diffusion-weighted imaging (DWI) and fluid-attenuated inversion recovery images were reviewed for $\mathrm{WMH}$, using the Cardiovascular Health Study (CHS) rating scale (ranging from 0 to 9 , with 9 being 'most extensive'). Linear regression was used to evaluate 'error rate on neglect test' as the dependent variable, as a measure of neglect severity,
\end{abstract}

with 'WMH category' as the primary independent variable, including adjustment for age, sex, race and infarct volume (on DWI). Logistic regression was used to evaluate a binary definition of neglect (defined as above) relative to the same independent variable and covariates. Results: Each 1-point increase in CHS leukoaraiosis category was associated with 1.20 -fold increased odds (95\% Cl: $1.00-1.43)$ of having any neglect, 1.23 -fold increased odds ( $95 \% \mathrm{Cl}: 1.02-1.49$ ) of having severe neglect and 1.33-fold increased odds of having worst neglect (95\% Cl: 1.01-1.76) after adjusting for infarct volume, age, sex and race. Increasing age and infarct size were also important predictors of neglect severity, with a $2.36 \%$ higher error rate ( $95 \% \mathrm{Cl}: 0.75-3.97 \%)$ on the line cancellation test associated with each category increase in CHS score; similar results were found for each of the neglect tests. Line cancellation neglect scores were worse in individuals with both severe WMH and large infarcts ( $p$ interaction, unadjusted $=0.03$ ). Conclusions: More severe leukoaraiosis is associated with more hemispatial neglect after acute ischemic stroke, independent of infarct volume, age and sex. We found not only more frequent neglect but also more severe neglect, based on error rates on neglect tests, in individuals with increasing leukoaraiosis. This emphasizes the importance of preexisting brain microvascular disease in outcomes of stroke patients. Further studies of the possible mechanism behind this association are needed.

(c) 2014 S. Karger AG, Basel

\section{KARGER}

E-Mail karger@karger.com

www.karger.com/ced (c) 2014 S. Karger AG, Basel

1015-9770/14/0373-0223\$39.50/0
Rebecca F. Gottesman, MD, $\mathrm{PhD}$

Department of Neurology, Johns Hopkins University School of Medicine, Phipps 446D

600 North Wolfe Street, Baltimore, MD 21287 (USA)

E-Mail rgottesm@jhmi.edu 


\section{Introduction}

Leukoaraiosis has been found to be strongly associated with cognitive decline and dementia longitudinally $[1,2]$, especially in patients with a history of stroke or transient ischemic attack [3]. Although the term 'leukoaraiosis' was selected to avoid inferences about the mechanism of these changes [4], it is currently understood to mean the changes observed on imaging to white matter tracts (white matter hyperintensities, WMH), and converging evidence supports at least a partial contribution from microvascular ischemic disease. In addition, more severe WMH are associated with higher risk of first-time and recurrent stroke $[5,6]$, and many risk factors such as age, hypertension and tobacco use are shared [7-9], with hypertension being the most important risk factor. It is not surprising, therefore, that many individuals who are hospitalized with acute ischemic stroke already have leukoaraiosis, or $\mathrm{WMH}$. In stroke patients, the presence of WMH has been associated with worse long-term outcomes $[10,11]$, death [12], lower quality of life [13] and worse outcome (hemorrhagic conversion) after tissue plasminogen activator (tPA) treatment [14] or thrombectomy [15].

The purpose of this study was to evaluate whether the presence and severity of WMH in right-hemisphere stroke patients was associated with worse cognitive performance, acutely after stroke, independent of infarct size. We evaluated prospectively admitted patients with acute ischemic right-hemispheric supratentorial stroke in order to evaluate whether extent of WMH on fluid-attenuated inversion recovery (FLAIR) imaging was associated with more frequent and more severe hemispatial neglect, independent of infarct volume.

We conducted an observational study on patients who were hospitalized for acute ischemic stroke and had cognitive assessment for neglect along with brain MRI. We hypothesized that people with a higher grade of WMH would have poor performance on cognitive tests (specifically, hemispatial neglect) after controlling for infarct volume.

\section{Methods}

\section{Patient Inclusion}

The study included patients admitted to Johns Hopkins Hospital and Johns Hopkins Bayview Medical Center from October 1999 through December 2011 for acute ischemic right-hemispheric stroke symptoms. The study was reviewed by the institutional review board, and informed consent was obtained prospectively from all participants. To be included, participants needed to be right-handed and have had neuropsychological assessment for hemispatial neglect within 5 days of symptom onset and within $24 \mathrm{~h}$ of hospitalization. Participants were excluded if they did not speak English, had a history of prior stroke, intracerebral hemorrhage or dementia, had uncorrected vision or hearing loss or had acute infratentorial infarcts on brain MRI. We included individuals with the neglect testing described below and a brain MRI, also within $24 \mathrm{~h}$ of admission and within 5 days of symptom onset.

\section{Cognitive Evaluation}

The assessment included a cognitive battery focused on assessment of hemispatial neglect, which consisted of line cancellation, line bisection (horizontal and vertical), oral reading and writing, Ogden copy scene, clock drawing as well as visual and tactile neglect tasks. For each patient, a Z score was calculated for each task. Neglect assessment was scored by trained research technicians without knowledge of imaging volumes or white matter scores. Z scores for neglect were defined based on normative data from 58 individuals admitted with transient ischemic attack or who were preoperative coronary artery bypass graft surgical candidates in other studies of cognition in these settings. We defined neglect both as a binary variable and as continuous error rates in specific neglect tests (we used those tests most frequently completed by the largest number of individuals). 'Any neglect' was defined as having a Z score of at least 2 on any of the following 3 tests: (1) horizontal line bisection, (2) line cancellation and (3) copying the Ogden scene [16]. These tests were selected because the data were the most complete on these (the tests were administered to the largest proportion of enrolled patients). 'Severe neglect' was defined as having a Z score of at least 2 on any 2 of those 3 tests, and 'worst neglect' as a $\mathrm{Z}$ score of at least 2 on all 3 of these tests (which required individuals to have completed all 3 tests). Error rates on each of these 3 tests were also evaluated, with higher scores indicating worse performance. In addition, the average error rate was evaluated as an additional measure of neglect severity, which was simply the mean of all 3 test scores, again with higher rates indicating worse performance.

\section{MRI Ratings}

Acute infarct volumes were measured manually by diffusionweighted imaging (DWI) using ImageJ software. Areas of acute infarct were traced on each DWI slice, and three-dimensional volumes were calculated based on these areas and slice thickness. We have previously reported our intraclass correlation coefficient for interrater reliability for these volumetric measurements by DWI, which is 0.978 [16]. Using the Cardiovascular Health Study (CHS) white matter rating scale [17], FLAIR images (standard 5-mm whole-brain axial slices) were reviewed by a single reviewer (Z.B.), without knowledge of the neglect scores, for WMH on a scale of $0-9$. The FLAIR images were compared with a set of templates demonstrating the CHS scale, displaying ratings ranging from 1 (minimal WMH) to 'severe and confluent white matter changes' (grade 8); studies without white matter changes were graded as 0 , and more extensive and worse than 8 were graded as 9 [17]. A subset of $10 \%$ of the FLAIR images were reviewed by a second investigator (R.G.). We observed $91.2 \%$ agreement for this subset, with a weighted $\kappa=0.68$. We defined 'severe' white matter changes as a CHS scale rating of 5 or higher. This represented approximately the top quartile of the white matter ratings. 


\section{Statistical Methods}

Stata version 13 for Macintosh (College Station, Tex., USA) was used for all analyses. Exploratory and descriptive analyses were conducted, and characteristics among individuals with severe (CHS score of 5 or higher) versus milder (including those with no $\mathrm{WMH}$ ) forms of WMH were compared using Pearson's $\chi^{2}$ tests (for categorical variables) and Student's t tests with unequal variances (for continuous variables).

Logistic regression models were created to evaluate the association between CHS white matter category with 'any neglect', including DWI infarct volume, age, sex and race as covariates. We evaluated CHS score both as an ordinal value and as a dichotomized value of severe ( 5 or higher) versus other WMH. We also performed linear regression models with the same predictors, but using error rate on each of the 3 primary neglect tests described above. Finally, to evaluate for an interaction between infarct volume and CHS WMH category - based on an a priori hypothesis that individuals with the combination of large volume infarct and more extensive $\mathrm{WMH}$ would do worse than individuals with either alone - we repeated the analyses, both with 'any neglect' as the dependent variable (logistic regression) as well as the linear regressions using error rates, but with the introduction of an interaction term, and also repeated analyses stratified by the quartile of DWI infarct volume.

Education levels were self-reported. We categorized them into 3 categories: $(1)<6$ th grade, $(2) 6$ th -12 th grade and $(3)>12$ th grade education. Information about the use of $\mathrm{tPA}$ for the stroke was also recorded.

\section{Sensitivity Analyses}

In order to determine whether our findings with error rates on the 3 neglect tests described above were due to the possibility of posterior cerebral artery involvement, we conducted a sensitivity analysis excluding all individuals with any errors on visual extinction testing (simultaneous stimulation). This would lead to the exclusion of individuals with both neglect and hemianopsia.

In another sensitivity analysis, we considered whether results would change if we entered the lowest level of education for those individuals with the neglect but with missing education data. We did this because there were a number of individuals missing education status.

\section{Results}

Of 308 potentially eligible participants who had some neglect testing and a brain MRI and presented with righthemispheric stroke symptoms, 12 were excluded for missing FLAIR or T2-weighted images, and therefore missing white matter grades, and 90 were excluded for missing 1 or 2 of the 3 neglect tests needed, leaving 206 participants for the final analyses using composite measures of neglect. Table 1 shows the characteristics of the individuals with severe WMH (CHS score $\geq 5$ ) compared with those with a CHS score $<5$. In univariate analyses, WMH score was highly associated with neglect perfor-
Table 1. Characteristics of individuals $(n=206)$ with mild versus severe WMH on brain MRI

\begin{tabular}{|c|c|c|c|}
\hline & $\begin{array}{l}\text { Milder } \\
\text { WMH } \\
(0-4) \\
(\mathrm{n}=148)\end{array}$ & $\begin{array}{l}\text { Severe } \\
\text { WMH } \\
(5-9) \\
(n=58)\end{array}$ & $\mathrm{p}$ \\
\hline Age, years & 58.9 & 71.5 & $<0.0001$ \\
\hline DWI volume, $\mathrm{cm}^{3}$ & 19.0 & 22.6 & 0.57 \\
\hline Sex - female, $\%$ & 44.9 & 48.3 & 0.66 \\
\hline Race - African-American, \% & 55.6 & 54.6 & 0.90 \\
\hline Patients with any neglect, ${ }^{\text {a }} \%$ & 35.8 & 58.6 & $<0.003$ \\
\hline Patients with severe neglect, ${ }^{\mathrm{b}} \%$ & 23.0 & 39.7 & $<0.016$ \\
\hline Line cancellation error rate, $\%$ & 10.4 & 20.8 & $<0.02$ \\
\hline Copy scene error rate, $\%$ & 17.0 & 32.2 & $<0.004$ \\
\hline Horizontal line bisection error rate, $\%$ & 5.3 & 9.0 & $<0.03$ \\
\hline NIHSS score & 4.5 & 5.7 & 0.16 \\
\hline Education level, ${ }^{\mathrm{c}} \%$ & & & 0.74 \\
\hline 6 th grade or lower & 5.6 & 10.0 & \\
\hline 6 th grade through high school & 60.8 & 60.0 & \\
\hline More than high school & 33.6 & 30.0 & \\
\hline
\end{tabular}

WMH ratings based on CHS white matter rating scale, ranging from 0 to 9 . $p$ values in italics are significant.

a 'Any neglect' defined as a Z score of at least 2 on any of the following tests: horizontal line bisection, line cancellation or copying the Ogden scene.

b 'Severe neglect' defined as a Z score of at least 2 on any 2 of the above 3 tests.

${ }^{\mathrm{c}}$ Education level only available on 145 of the participants.

mance. There was a nearly linear increase in odds of any of the neglect definitions (any, severe or worst) as the CHS category increased (fig. 1).

In multivariable analyses (table 2), an increasing CHS category (by 1 point) was associated with 1.20 -fold increased odds of having any neglect (95\% CI: 1.00-1.43), 1.23 -fold increased odds of having severe neglect $(95 \%$ CI: 1.02-1.49) and 1.33-fold increased odds of having worst neglect (95\% CI: 1.01-1.76). These values were all adjusted for DWI volume, age, gender and race. Within these models, volume and age were also independent predictors of any neglect. Although the p interaction for age and $\mathrm{WMH}$ was not significant (with each dichotomized, with age at 65 years and $\mathrm{WMH}$ at 5 and higher vs. below $5)$, there was a trend toward worse neglect performance (higher error rates, suggesting more severe neglect) in individuals with older age and more WMH (fig. 2).

Table 3 shows similar results when error rates on each of the test scores were used individually (adjusted for age, race, gender and infarct volume). Beta value, as an incremental change in error rate for each test, was associated 
Fig. 1. Probability of having 'any neglect' (a) Z score of at least 2 on any of these 3 tests: line cancellation, Ogden copy scene, horizontal line bisection) or 'severe neglect' ( $\mathbf{b}$; $Z$ score of at least 2 on any 2 of the above 3 tests), dependent on the CHS white matter rating scale $(0-9$; 9 being most severe). Crude odds ratios for having 'any neglect' are listed for each CHS category, compared with a rating of 0 as the reference group (Ref). OR = Odds ratio.

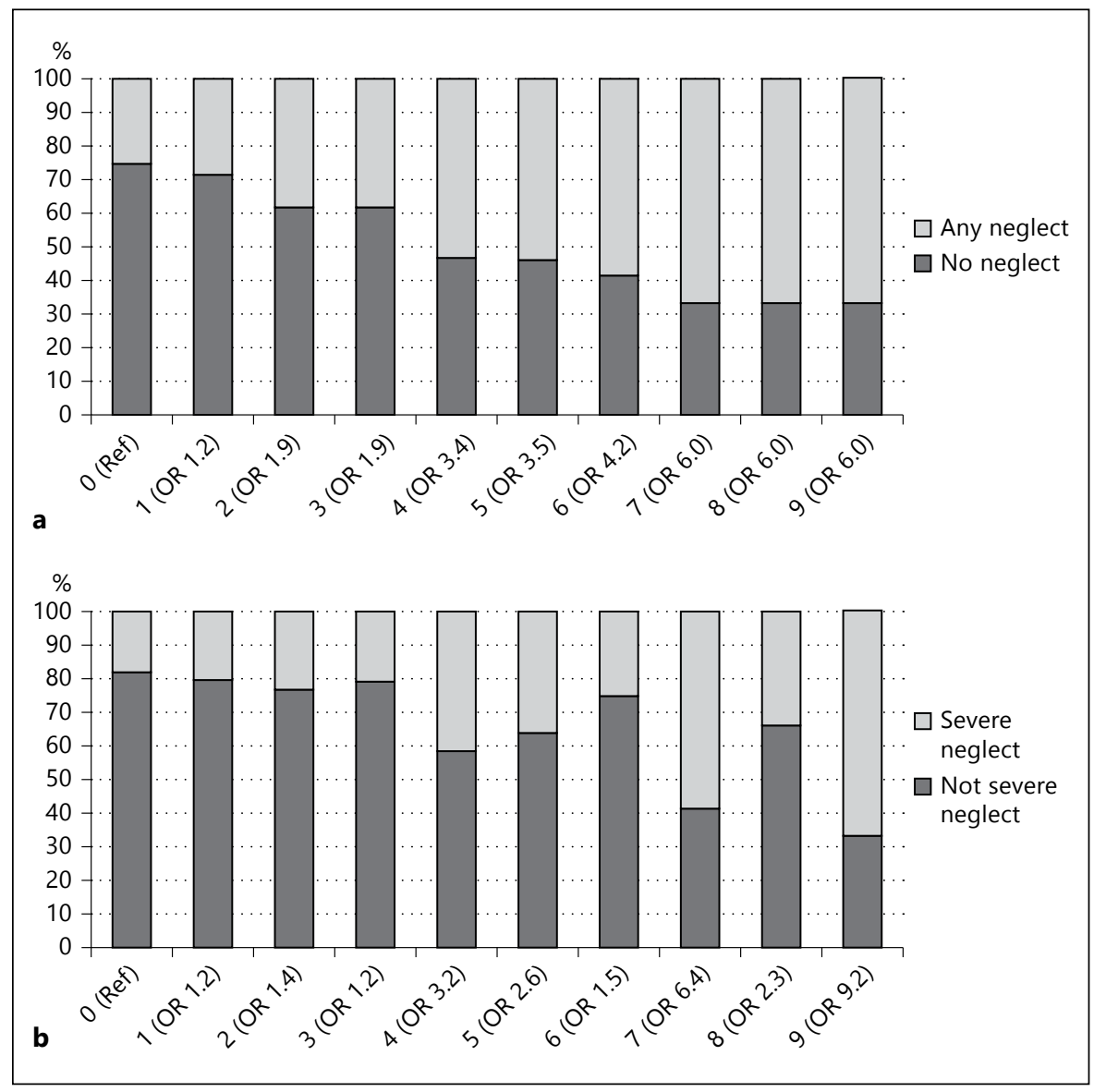

Table 2. Multivariable odds ratios for each type of neglect

\begin{tabular}{|c|c|c|c|}
\hline & Any neglect & Severe neglect & Worst neglect \\
\hline CHS white matter category (per 1-point higher category) & $1.20(1.00-1.43)$ & $1.23(1.02-1.49)$ & $1.33(1.01-1.76)$ \\
\hline Infarct volume (per $10 \mathrm{~cm}^{3}$ ) measured by DWI & $1.68(1.36-2.09)$ & $1.53(1.30-1.80)$ & $1.27(1.15-1.41)$ \\
\hline Sex (female vs. male) & $1.74(0.85-3.55)$ & $2.29(1.03-5.11)$ & $0.67(0.22-2.04)$ \\
\hline Race (African-American vs. Caucasian or other) & $0.79(0.38-1.63)$ & $1.20(0.54-2.68)$ & $1.35(0.44-4.13)$ \\
\hline
\end{tabular}

Values in parentheses denote 95\% CI. All variables were in the same model, with 3 separate models (1 for any neglect, 1 for severe neglect and 1 for worst neglect). Values in bold indicate those reaching statistical significance $(\mathrm{p} \leq 0.05)$.

as being 1 category higher on the CHS white matter rating scale. Dichotomizing the white matter category variable (CHS scale score $0-4$ vs. 5-9) was associated with a trend toward increased odds of any neglect or severe neglect [adjusted odds ratio of 1.96 for any neglect (95\% CI: 0.90 4.26) for individuals with CHS scale scores of 5-9 vs. a lower value, for severe neglect] but with significantly in- creased odds of having worst neglect (adjusted odds ratio: 3.42 ; 95\% CI: 1.00-11.63). Each increasing quartile on the CHS scale was associated with, on average, a $6.15 \%$ higher error rate on the line cancellation task (95\% CI: 2.96$9.34 \%$ ), a $5.14 \%$ higher error rate on the copy scene task (95\% CI: $1.78-8.50 \%)$ and a $1.95 \%$ higher error rate $(95 \%$ CI: $0.58-3.32 \%)$ on horizontal line bisection, after adjust- 


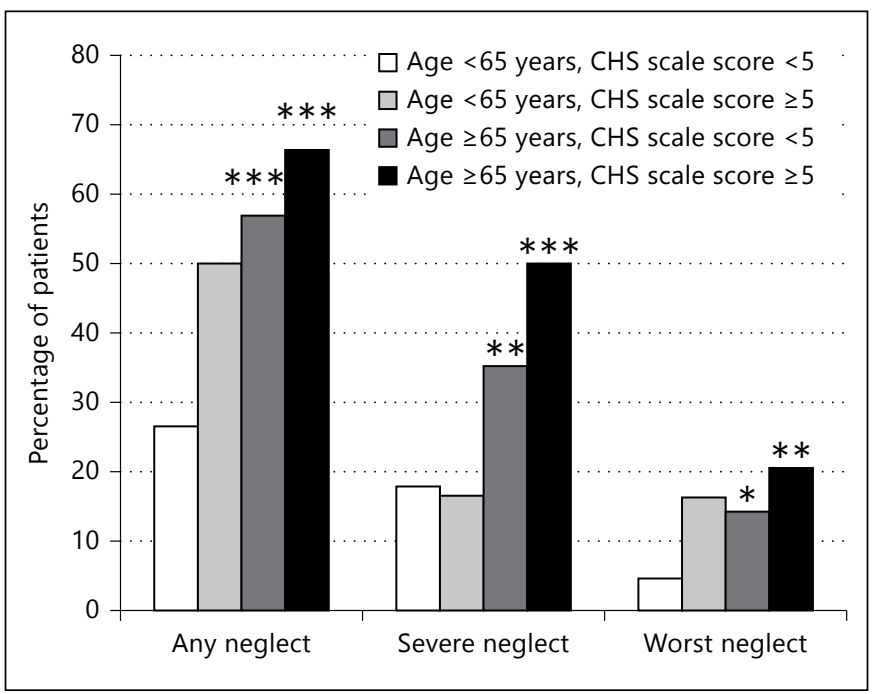

Fig. 2. Frequency of different types of neglect among patients as determined by age group and $\mathrm{WMH}$ status (using CHS rating scale). ${ }^{*} \mathrm{p}<0.05,{ }^{* *} \mathrm{p}<0.01$ and ${ }^{* * *} \mathrm{p}<0.001$ when compared with bar with youngest age and lowest CHS category.

Table 3. Beta coefficients for error rates on neglect tests (with higher numbers indicating worse performance), per 1 category higher CHS white matter rating scale rating (range: $0-9$, with higher category indicating more severe $\mathrm{WMH}$ )

\begin{tabular}{llll}
\hline & Beta & $95 \%$ CI & $\mathrm{p}$ \\
\hline Line cancellation & 2.36 & $0.75-3.97$ & 0.004 \\
Copy scene & 2.53 & $0.81-4.24$ & 0.004 \\
Horizontal line bisection & 0.93 & $0.26-1.61$ & 0.007 \\
\hline
\end{tabular}

Regression coefficients are adjusted for age, sex, race and DWI infarct volume.

ment for age, gender and infarct size. The mean error rate was also strongly predicted by the extent of $\mathrm{WMH}$, independent of age, sex, race and infarct size [beta $=1.94$ (95\% CI: 0.79-3.08) per 1-point-higher CHS category]; WMH $\geq 5$ was associated with, on average, an 8.9-point-higher mean error rate, independent of these same variables (95\% CI: 2.30-15.51). The results were similar when tPA status (used in 9 patients) was included as a covariate.

Addition of educational level to these models led to similar results but with attenuation of results, with smaller sample sizes due to missing education data on approximately $30 \%$ of the sample and therefore larger standard errors. The analyses of mean error rate and horizontal line bisection remained statistically significant, with loss of statistical significance $(p=0.071,0.063)$ for the models evaluating Ogden copy scene and line cancellation scores, with similar coefficients. The logistic regression models for 'any neglect' and other neglect types did not remain statistically significant.

In addition to the primary associations described above, a test for interaction between DWI volume and $\mathrm{WMH}$ ratings neared significance for the line cancellation task $(\mathrm{p}=0.06)$. We did not observe a statistically significant interaction, however, for 'any neglect' as a dichotomized variable between DWI volume and WMH category.

\section{Sensitivity Analysis}

When we excluded individuals with any errors on visual extinction double simultaneous stimulation testing, we found that results were unchanged despite the large reduction in sample size (since many individuals with neglect on the 3 primary tests included also had visual extinction).

When analyses were repeated with education adjustment but with a replacement of a missing educational value with the lowest level of education for those individuals with neglect, results were similar to the primary results without adjustment for education (similar effect sizes and statistical significance).

\section{Discussion}

Our study of hospitalized stroke patients with righthemispheric stroke symptoms demonstrates that the extent of preexisting WMH may be important in the clinical manifestation of stroke symptoms. Evidence for a true physiologic relationship is further strengthened by the increasing frequency and severity of neglect as the grade of $\mathrm{WMH}$ increases. This is important because $\mathrm{WMH}$ are typically thought to involve subcortical processing deficits, as opposed to cortically derived deficits, such as neglect (right hemisphere) or language (left hemisphere). Figure 3 shows 2 examples of patients with similar infarct volumes but very different white matter grades and more severe neglect performance on the copy scene task and the line cancellation task for the individual with highergrade $\mathrm{WMH}$. We also found that increasing age remains a risk factor for neglect, as we have previously reported [16], as does infarct volume. Increasing age and higher levels of WMH appear to be associated with even worse neglect than with either of those factors alone, although 


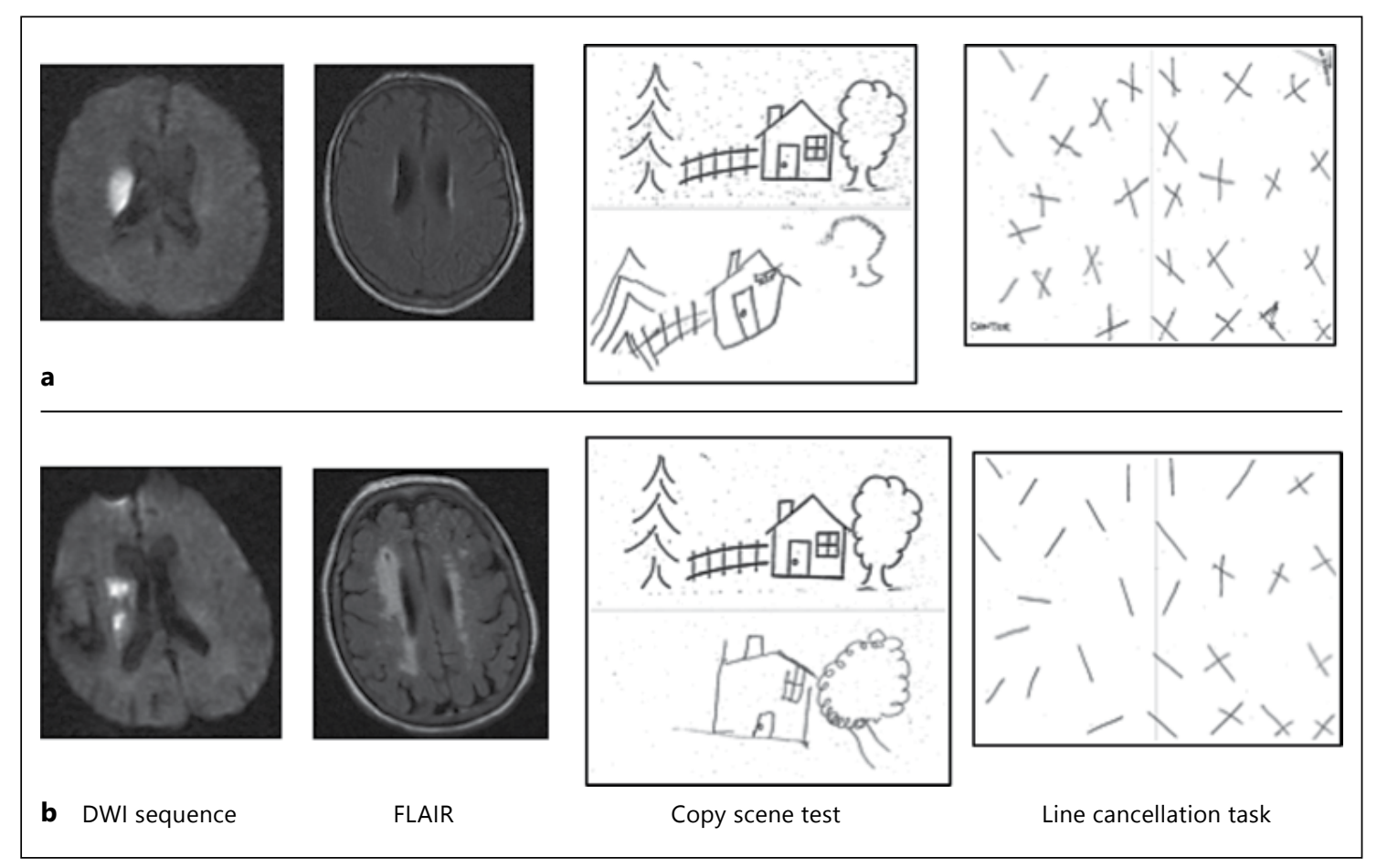

Fig. 3. Two patient examples demonstrating DW images with small, similar-sized infarcts. FLAIR sequences showing low- (participant A; a) and high-grade (participant B; b) WMH, and copy scene and line cancellation tasks demonstrating worse neglect with poor performance for participant B (b) relative to participant A (a).

the sample size was not adequate to demonstrate a statistical interaction between age and WMH.

Although other studies have demonstrated the importance of preexisting microvascular disease, including $\mathrm{WMH}$, in the long-term outcome of individuals with stroke $[10,11,18]$, few studies provide a possible mechanism. Although this study does not provide a specific mechanism, it suggests that the impact of WMH is early (immediately after a stroke) and is relevant to cognitive functions otherwise thought to be primarily due to cortical injury. Neglect is most commonly associated with cortical lesions [19], although the role of lesions to white matter tracts is also recognized [20-22]. WMH are subcortical, and are usually associated with more global cognitive dysfunction [23]. Specifically, it is expected that $\mathrm{WMH}$ would have an impact on nonspatial attention, motor speed and executive function [1,24]. Neglect is often considered a two-component deficit: (1) an imbalance in laterally biased spatial attention (which results from damage to the dorsal attention system in either hemisphere) and (2) disruption in nonspatially biased attention such as vigilance and selective attention; the latter most likely results from damage to the right hemi- sphere-dominant ventral attention system, which plausibly depends on white matter tracts connecting the temporal-parietal junction to the frontal cortices [2527].

We did not separate out cortical from subcortical infarcts in our study, but anticipate that some of those individuals with purely subcortical infarcts may have had cortical hypoperfusion. We do not anticipate, however, that analyzing those with cortical versus subcortical strokes would change the relationship between WMH and neglect. Individuals with cortical involvement would probably have more severe neglect in general, but this should not have a differential impact on the role of WMH.

A potential mechanism of WMH leading to worse cognitive performance after stroke is that compensation and plasticity of recovery after stroke might be adversely affected by impaired subcortical structures. Reorganization of function occurs after stroke, and cortical connectivity between different brain regions through white matter tracts may be critical to the recovery and redistribution of function [28]. The fact that the association was found to be strong, independent of DWI infarct volume, indicates that the findings observed cannot be attributed to an as- 
sociation between extensive white matter disease and larger strokes (since infarct volume is an independent predictor of neglect in our series). Our data suggest that individuals with preexisting WMH may be at higher risk of worse cognitive outcomes acutely after stroke, and thus might benefit the most from early aggressive rehabilitation. It is therefore critical that the relationship between white matter changes and stroke rehabilitation, from the acute stages to chronic recovery, is further clarified.

Our study is limited by the fact that WMH are rated based on MRI scans performed after the stroke. It is therefore difficult to be certain that the WMH excluded cytotoxic edema from the stroke itself. However, most of the $\mathrm{WMH}$ were symmetric, which would not be consistent with these being due to stroke-related edema. We also are limited in our assessment of the pattern of the WMH; we used a standardized scale, the CHS rating scale, which does not separate periventricular and subcortical WMH or include any regional description of WMH distribution. It is a relatively qualitative scale, and not a quantitative volumetric measurement. Similarly, our volumetric measurements of infarcts, measured using ImageJ, are potentially susceptible to measurement error. We do not, however, anticipate any bias in the error, since the same technique was applied to all scans and the individuals measuring the scans were blinded to $\mathrm{WMH}$ volumes and neglect scores. In addition, to generate our composite definitions of 'any neglect' or 'severe neglect', individuals required data on 2 or 3 of the tests included. Individuals missing data on all 3 were older and had larger strokes, which means our included population may be missing the more severe cases of neglect or at least the larger strokes. However, results were similar when individual test scores were evaluated, with more severe WMH associated with higher error rates on all 3 tests individually, in adjusted models. The addition of education to the models led to attenuation of some of the associations observed, likely due to missing data and therefore larger standard errors. Because of the assumption that less-educated people might have more neglect, our sensitivity analysis imputing low education levels for people with neglect confirmed our primary analyses.

Our data support the existing literature indicating that prestroke brain disease, such as WMH, may adversely affect an individual's ability to recover from stroke. In addition, they add the new information that specific cognitive functions - acutely impaired in stroke - may be worse independent of infarct volume in the presence of more severe WMH. These results suggest that recovery is not fully determined by an acute stroke lesion alone, but rather by a preexisting brain disease that mitigates poststroke cognitive performance.

\section{Acknowledgements}

This study was supported by the National Institutes of Health (grant R01 NS047691 to A.B.H.).

\section{Disclosure Statement}

The authors have no conflicts of interest to report.

\section{References}

1 Jokinen H, Kalska H, Ylikoski R, Madureira S, Verdelho A, Gouw A, Scheltens P, Barkhof F, Visser MC, Fazekas F, Schmidt R, O’Brien J, Hennerici M, Baezner H, Waldemar G, Wallin $\mathrm{A}$, Chabriat $\mathrm{H}$, Pantoni L, Inzitari $\mathrm{D}$, Erkinjuntti T; LADIS Group: MRI-defined subcortical ischemic vascular disease: baseline clinical and neuropsychological findings. The LADIS Study. Cerebrovasc Dis 2009;27: 336-344.

2 Carmichael O, Schwarz C, Drucker D, Fletcher E, Harvey D, Beckett L, Jack CR Jr, Weiner M, DeCarli C; Alzheimer's Disease Neuroimaging Initiative: Longitudinal changes in white matter disease and cognition in the first year of the Alzheimer disease neuroimaging initiative. Arch Neurol 2010;67:1370-1378.

-3 Dufouil C, Godin O, Chalmers J, Coskun O, MacMahon S, Tzourio-Mazoyer N, Bousser MG, Anderson C, Mazoyer B, Tzourio C;
PROGRESS MRI Substudy Investigators: Severe cerebral white matter hyperintensities predict severe cognitive decline in patients with cerebrovascular disease history. Stroke 2009;40:2219-2221.

4 Hachinski VC, Potter P, Merskey H: Leukoaraiosis. Arch Neurol 1987;44:21-23.

5 Vermeer SE, Hollander M, van Dijk EJ, Hofman A, Koudstaal PJ, Breteler MM: Silent brain infarcts and white matter lesions increase stroke risk in the general population. Stroke 2003;34:1126-1129.

-6 Melkas S, Sibolt G, Oksala NK, Putaala J, Pohjasvaara T, Kaste M, Karhunen PJ, Erkinjuntti $\mathrm{T}$ : Extensive white matter changes predict stroke recurrence up to 5 years after a first-ever ischemic stroke. Cerebrovasc Dis 2012;34:191-198.

7 Prabhakaran S, Wright CB, Yoshita M, Delapaz R, Brown T, DeCarli C, Sacco RL: Preva- lence and determinants of subclinical brain infarction. Neurology 2008;70:425-430.

-8 van Dijk EJ, Prins ND, Vrooman HA, Hofman A, Koudstaal PJ, Breteler MM: Progression of cerebral small vessel disease in relation to risk factors and cognitive consequences. Stroke 2008;39:2712-2719.

-9 Gottesman RF, Coresh J, Catellier DJ, Sharrett AR, Rose KM, Coker LH, Shibata DK, Knopman DS, Jack CR, Mosley TH Jr: Blood pressure and white matter disease progression in a biethnic cohort: Atherosclerosis Risk in Communities (ARIC) study. Stroke 2010; 41:3-8.

10 Arsava EM, Rahman R, Rosand J, Lu J, Smith EE, Rost NS, Singhal AB, Lev MH, Furie KL, Koroshetz WJ, Sorensen AG, Ay H: Severity of leukoaraiosis correlates with clinical outcome after ischemic stroke. Neurology 2009; 72:1403-1410.
White Matter Changes and Neglect in Stroke Patients
Cerebrovasc Dis 2014;37:223-230 DOI: $10.1159 / 000357661$ 
-11 Henninger N, Lin E, Baker SP, Wakhloo AK, Takhtani D, Moonis M: Leukoaraiosis predicts poor 90-day outcome after acute large cerebral artery occlusion. Cerebrovasc Dis 2012;33:525-531.

12 Conjin M, Kloppenberg RP, Algra A, Mali WP, Kappelle LJ, Vincken KL, van der Graaf Y, Geerlings MI; SMART Study Group: Cerebral small vessel disease and risk of death, ischemic stroke, and cardiac complications in patients with atherosclerotic disease: the Second Manifestations of ARTerial DiseaseMagnetic Resonance (SMART-MR) study. Stroke 2011;42:3105-3109.

13 Koton S, Schwammenthal Y, Merzeliak O, Philips T, Tsabari R, Orion D, Dichtiar R, Tanne D: Cerebral leukoaraiosis in patients with stroke or TIA: clinical correlates and 1-year outcome. Eur J Neurol 2009;16:218225.

14 Aries MJ, Uyttenboogaart M, Vrooman PC, de Keyser J, Luijckx GJ: tPA treatment for acute ischaemic stroke in patients with leukoaraiosis. Eur J Neurol 2010;17:866-870.

15 Shi ZS, Loh Y, Liebeskind DS, Saver JL, Gonzalez NR, Tateshima S, Jahan R, Feng L, Vespa PM, Starkman S, Salamon N, Villablance JP, Ali LK, Ovbiagele B, Kim D, Viñuela F, Duckwiler G: Leukoaraiosis predicts parenchymal hematoma after mechanical thrombectomy in acute ischemic stroke. Stroke 2012;43. 1806-1811.
6 Gottesman RF, Kleinman JT, Davis C, Heidler-Gary J, Newhart M, Kannan V, Hillis AE: Unilateral neglect is more severe and common in older patients with right hemispheric stroke. Neurology 2008;71:14391444.

17 Manolio TA, Kronmal RA, Burke GL, Poirier V, O'Leary DH, Gardin JM, Fried LP, Steinberg EP, Bryan RN: Magnetic resonance abnormalities and cardiovascular disease in older adults. The Cardiovascular Health Study. Stroke 1994;25:318-327.

18 Putaala J, Haapaniemi E, Kurkinen M, Salonen O, Kaste M, Tatlisumak T: Silent brain infarcts, leukoaraiosis, and long-term prognosis in young ischemic stroke patients. Neurology 2011;76:1742-1749.

19 Ringman JM, Saver JL, Woolson RF, Clarke WR, Adams HP: Frequency, risk factors, anatomy, and course of unilateral neglect in an acute stroke cohort. Neurology 2004;63: 468-474.

20 Bartolomeo P: Visual neglect. Curr Opin Neurol 2007;20:381-386.

21 Doricchi F, Thiebaut de Schotten M, Tomaiuolo F, Bartolomeo P: White matter (dis)connections and gray matter (dys)functions in visual neglect: gaining insights into the brain networks of spatial awareness. Cortex 2008; 44:983-995.

22 Umarova RM, Saur D, Schnell S, Kaller CP, Vry MS, Glauche V, Rijntjes M, Hennig J, Kiselev V, Weiller C: Structural connectivity for visuospatial attention: Significance of ventral pathways. Cereb Cortex 2010;20:121129.
23 Schmidt R, Berghold A, Jokinen H, Gouw AA, van der Flier WM, Barkhof F, Scheltens P, Petrovic K, Madureira S, Verdelho A, Ferro JM, Waldemar G, Wallin A, Wahlund LO, Poggesi A, Pantoni L, Inzitari D, Fazekas F, Erkinjuntti T; LADIS Study Group: White matter lesion progression in LADIS: frequency, clinical effects, and sample size calculations. Stroke 2012;43:2643-2647.

24 Prins ND, van Dijk EJ, den Heijer T, Vermeer SE, Jolles J, Koudstaal PJ, Hofman A, Breteler MM: Cerebral small-vessel disease and decline in information processing speed, executive function and memory. Brain 2005;128: 2034-2041.

25 Corbetta M, Shulman GL: Control of goal-directed and stimulus-directed attention in the brain. Nat Rev Neurosci 2002;3:201-216.

26 Corbetta M, Shulman GL: Spatial neglect and attention networks. Annu Rev Neurosci 2011; 34:569-599.

27 Thiebaut de Schotten M, Tomaiuolo F, Aiello M, Merola S, Silvetti M, Lecce F, Bartolomeo P, Doricchi F: Damage to white matter pathways in subacute and chronic spatial neglect: a group study and 2 single-case studies with complete virtual 'in vivo' tractography dissection. Cereb Cortex 2012, Epub ahead of print.

28 Grefkes C, Fink GR: Reorganization of cerebral networks after stroke: new insights from neuroimaging with connectivity approaches. Brain 2011;134:1264-1276. 